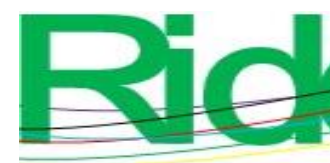

\title{
Disimetrías de las investigadoras en los centros de investigación del Instituto Politécnico Nacional. Un estudio cuantitativo
}

\author{
Researchers' Asymmetries in the Research Centers of the Instituto \\ Politécnico Nacional. A Quantitative Study
}

\section{Dissimetrias dos pesquisadores nos centros de pesquisa do Instituto Politécnico Nacional. Um estudo quantitativo}

\author{
José de Jesús Peinado Camacho \\ Instituto Politécnico Nacional, México \\ jpeinadoc@ipn.mx \\ https://orcid.org/0000-0002-2262-4565
}

\section{Resumen}

El objetivo de la presente investigación fue encontrar y analizar datos cuantitativos que muestren las disimetrías de las investigadoras en los centros de investigación del Instituto Politécnico Nacional (IPN). El diseño de la investigación fue no experimental y transeccional. La metodología fue cuantitativa con enfoque exploratorio y descriptivo. Los resultados del estudio permitieron conocer que en los centros de investigación del IPN el número de investigadoras es menor al de investigadores. Asimismo, distribuidos según su nivel de preparación, hay más hombres que mujeres en todos los grados educativos. En cuanto al tipo de contratación, el personal masculino predominó en todos los apartados, a excepción de lo registrado en la casilla de 30 horas, donde las cantidades fueron semejantes. Con respecto a las becas y estímulos que otorgó el IPN, Licencias con Goce de Sueldo es el rubro con mayor número de investigadoras; en todos los demás el personal masculino registra arriba de $50 \%$. En relación con los miembros del Sistema Nacional de Investigadores (SNI), 


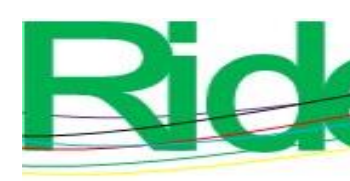

Revista Iberoamericana para la Investigación y el Desarrollo Educativo ISSN 2007 - 7467

hay menos investigadoras que investigadores y se registra un desequilibrio aún mayor conforme el nivel es más alto. En los proyectos de investigación, hay una menor participación de mujeres. La recopilación de datos de los colegios de profesores permitió evidenciar que solamente cuatro centros de investigación tuvieron $50 \%$ o más de investigadoras. La participación de las investigadoras en las redes de investigación del instituto fue igualmente baja. En cuanto a las redes de género, por el contrario, fue escasa la colaboración del personal masculino. Los datos reafirman el reflejo de las disimetrías que enfrentan las investigadoras. Los datos numéricos exhibieron pocos avances desde la perspectiva de género.

Palabras clave: centro de investigación, disimetrías, investigadoras, perspectiva de género.

\section{Abstract}

The objective of the research was to find and analyze quantitative data that shows the asymmetries of the female researchers in the research centers of the Instituto Politécnico Nacional (IPN). The research design was non-experimental and transectional. The methodology was quantitative with an exploratory and descriptive approach. The results of the study revealed that in the research centers of the IPN the number of female researchers is less than the number of male researchers. Regarding the type of hiring, male staff predominated in all sections, except for what was registered in the 30-hour category, where the amounts were similar. With regard to the scholarships and incentives granted by the IPN, paid leave was the only section in which a greater number of female researchers was perceived; in all the rest, the male staff rose above $50 \%$. In relation to the members of the National System of Researchers (SNI, by its acronym in Spanish), there are fewer female researchers than male researchers and an even greater imbalance is recorded as the level is higher. Less research participation prevailed in research projects. The collection of data from teacher colleges revealed that only four research centers had $50 \%$ or more female researchers. The participation of the researchers in the institute's research networks was low. Regarding gender networks, there was little collaboration from male staff in this activity. It was concluded that the data reaffirms the reflection of the asymmetries that the female researchers face. Numerical data showed little progress from a gender perspective.

Keywords: research center, asymmetries, researchers, gender perspective. 


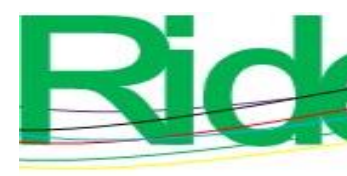

Revista Iberoamericana para la Investigación y el Desarrollo Educativo ISSN $2007-7467$

\section{Resumo}

O objetivo desta pesquisa foi encontrar e analisar dados quantitativos que evidenciam as assimetrias dos pesquisadores nos centros de pesquisa do Instituto Politécnico Nacional (IPN). O desenho da pesquisa foi não experimental e transversal. A metodologia foi quantitativa com abordagem exploratória e descritiva. Os resultados do estudo revelaram que o número de pesquisadores nos centros de pesquisa do IPN é menor que o número de pesquisadores. Além disso, distribuído de acordo com o nível de preparação, há mais homens do que mulheres em todos os níveis de escolaridade. Quanto ao tipo de contratação, predominou o pessoal do sexo masculino em todos os setores, exceto no que constava do quadro 30 horas, onde os valores foram semelhantes. Em relação às bolsas e incentivos concedidos pelo IPN, Licenças com Remuneração é a categoria com maior número de pesquisadores; nas demais, o efetivo masculino é superior a 50\%. Em relação aos membros do Sistema Nacional de Pesquisadores (SNI), há menos pesquisadoras do que pesquisadores do sexo masculino e um desequilíbrio ainda maior se registra à medida que o nível é maior. Em projetos de pesquisa, há menos participação de mulheres. A coleta de dados nas escolas de professores mostrou que apenas quatro centros de pesquisa tinham $50 \%$ ou mais pesquisadoras. A participação de pesquisadoras nas redes de pesquisa do instituto foi igualmente baixa. Em relação às redes de gênero, ao contrário, houve pouca colaboração do pessoal masculino. Os dados reafirmam o reflexo das assimetrias enfrentadas pelos pesquisadores. Os dados numéricos mostraram pouco progresso do ponto de vista de gênero. Palavras-chave: centro de pesquisa, assimetrias, pesquisadores, perspectiva de gênero. Fecha Recepción: Abril 2020 Fecha Aceptación: Septiembre 2020

\section{Introducción}

Izquierdo y Atristan (2019) señalan que "las investigadoras se enfrentan a diversas tensiones en su labor, el ritmo de la vida académica desplaza el cuidado de sí mismas, de su salud y de sus espacios de esparcimiento y de disfrute" (p. 138). Aunado a esto, se encuentran la maternidad, los hijos, el cuidado de sus padres, la pareja, la edad, la división sexual del trabajo y otras más (Blazquez y Bustos, 2013, p. 147; Buquet, 2011, p. 222; Gómez y Tena, 2018, p. 1). Se trata de dificultades muy difíciles de solventar. 


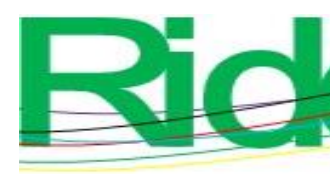

Revista Iberoamericana para la Investigación y el Desarrollo Educativo ISSN 2007 - 7467

Para Cárdenas (2015), "las mujeres investigadoras han carecido de oportunidades igualitarias en el mercado de trabajo" (p. 9). McDonald (2016) refiere que "aún la segregación ocupacional por formas de diferencia socialmente significativas como el sexo y la raza se encuentran presentes" (p. 19). En este sentido, las categorías sociales se relacionan con las desigualdades de manera compleja (Heiskanen, Otonkorpi, Leinonen y Ylöstalo, 2018, p. 459). Todavía permanece la creencia de una división natural del trabajo entre mujeres y varones debido a la diferencia sexual (Bustos, 2017, p. 288; Gamboa y Pérez, 2017, p. 256). Incluso se siguen asignando imágenes de valor a las prácticas de colaboración en formas estereotipadas de género (Castellano, 2 de julio de 2019, p. 35; Zippel, 2019, p. 1794), lo que predispone la exclusión de las investigadoras (Nielsen, 2017, p. 151).

En esa misma línea, Sinha (2015) expone que "la participación de las mujeres en el empleo remunerado en Japón, China e India, han carecido de oportunidades igualitarias a pesar de ser las tres economías más grandes de Asia y de la gran expansión de acceso a la educación en los últimos cincuenta años” (p. 846). Por su parte, Nielsen (2017) relata que “en Dinamarca persisten las estratificaciones académicas de género, un país reconocido por su posición internacional en temas de igualdad social de género” (p. 134). Gupta (2016) explica que "en la India el patriarcado y la jerarquía contribuyen a crear un ambiente masculino en el lugar de trabajo de las mujeres científicas” (p. 437). Estrada, Mendieta y González (2016) describen que “en México se mantienen y se reproducen estereotipos, roles y prejuicios que promueven la discriminación y el trato desigual, producto de la cultura e idiosincrasia" (p. 12).

La vida laboral engendra desigualdades en la sociedad, como son oportunidades económicas (Jabbaz, Samper y Díaz, 2019, p. 6) y expectativas de carrera (García, 2014, p. 22; Heiskanen et al., 2018, p. 459). Mattsson (2015) subraya que "la desigualdad de género en el mundo académico es un efecto de la creencia de contradicción entre la mujer y la ciencia" (p. 685). Esto es influenciado, en gran parte, por la idealización de los roles familiares de las mujeres (Nikunen y Lempiäinen, 2018, p. 554), lo que afecta su posición como científicas (Gupta, 2016, p. 437), de tal manera que las variables asociadas con la familia pueden ser parte de prácticas discriminatorias estructurales y sistémicas (Aiston y Jung, 2015, p. 205); ahí, en el núcleo familiar, persiste la centralidad del matrimonio y la crianza de los hijos, pero no el trabajo remunerado (Moreno, 2018, p. 140; Sinha, 2015, p. 846). 


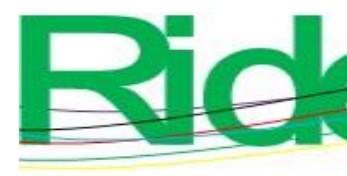

Revista Iberoamericana para la Investigación y el Desarrollo Educativo

ISSN $2007-7467$

Blazquez y Bustos (2013) advierten que "aunadas a las condiciones de desarrollo de las académicas, se encuentran los puestos de toma de decisión que llegan a convertirse en un obstáculo” (p. 147), ocupados principalmente por personal masculino (Nielsen, 2017, p. 149), y en ocasiones usados como herramienta de desigualdad y segregación (Blanco, 2018, p. 11). Al respecto, es necesario decir que las dinámicas de poder están presentes en la investigación, así como en los roles de representación, el posicionamiento de las investigadoras y la identidad de género (Hoskins, 2015, p. 393; Kannen, 2013, p. 178). Adicionalmente, Nielsen (2017) establece que "los conocimientos sobre las condiciones estructurales y culturales que circunscriben las ambiciones profesionales y las expectativas de las investigadoras, se unen en un punto de transición resumido por las altas demandas de productividad académica" (p. 149). Las estructuras de recompensa institucional unen valores simbólicos y materiales de género (García, 2014, p. 22; Moreno, 2018, p. 164; Zippel, 2019, p. 1794).

Por otra parte, Macías e Islas (2018) establecen que "las tecnologías de la información y la comunicación [TIC] representan ventajas para las labores de investigación que realizan las mujeres, pero aún no son percibidas como un instrumento de empoderamiento" (p. 208). De acuerdo con Gamboa y Pérez (2017), con el desarrollo de las TIC "se resuelve el problema de movilidad pero no la dificultad del tiempo para el trabajo, para la familia o el tiempo personal” (p. 265). Bustos (2017) añade que "el uso del tiempo es determinante en la construcción de las trayectorias laborales; las académicas encuentran limitaciones para desarrollar su trayectoria laboral, por la divergencia de distribuir su tiempo entre el trabajo no remunerado y el trabajo remunerado” (p. 269). Meza, Galbán y Ortega (2019) concluyen que "el reto es el balance de la vida familiar y el trabajo" (p. 29).

\section{EI Instituto Politécnico Nacional}

El Instituto Politécnico Nacional (IPN) cuenta con 100 unidades. En los tres niveles educativos que se imparten en las modalidades escolarizada, no escolarizada y mixta, participan más de 420000 personas. Tiene presencia en 32 localidades de 21 entidades federativas de México, a través de 19 unidades académicas del nivel medio superior, 27 unidades académicas del nivel superior, 20 centros de investigación científica y tecnológica, 17 centros de educación continua, cuatro unidades de apoyo educativo, tres unidades de apoyo a la innovación educativa, ocho unidades de apoyo a la investigación, al desarrollo y 


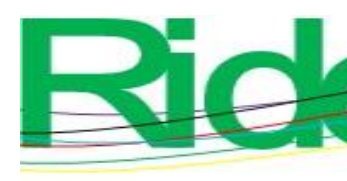

Revista Iberoamericana para la Investigación y el Desarrollo Educativo ISSN 2007 - 7467

fomento tecnológico y empresarial y dos unidades educativas vinculadas a ciencia, tecnología, investigación y desarrollo empresarial. Al conjuntar los tres niveles educativos, brinda una oferta educativa de 262 programas académicos. Asimismo, cuenta con una matrícula de 178492 alumnos, cifra a la que si todavía se le suman los servicios de educación continua e idiomas arroja un total de 390000 personas atendidas anualmente. En lo que respecta a la investigación, el IPN cuenta con personal académico de calidad que se refleja en los 1216 profesores que se encuentran registrados en el Sistema Nacional de Investigadores (SNI), lo que lo posiciona en el segundo lugar a nivel nacional, sin contar lo correspondiente al Centro de Investigación y de Estudios Avanzados (IPN, 2018, pp. 19-31)

De acuerdo con el Reglamento Orgánico del IPN (2020):

Los centros de investigación científica y tecnológica son unidades académicas que se encargan de realizar investigación básica, aplicada y desarrollo tecnológico, orientando sus resultados a la solución de problemas en áreas estratégicas del desarrollo nacional, para generar conocimientos que contribuyan en el avance de la ciencia y la tecnología, a la formación de recursos humanos de alto nivel, a mejorar la calidad de vida de la población, satisfacer necesidades específicas de los sectores público, social o privado y a la conservación y uso sustentable de los recursos naturales del país (p. 58).

El tópico de perspectiva de género ha sido poco estudiado de forma directa en los centros de investigación del IPN. Por tal motivo, aquí se acoge precisamente como objeto de estudio. La meta fue encontrar y analizar datos cuantitativos que muestren las disimetrías de las investigadoras en estos centros de investigación científica y tecnológica.

La pregunta de investigación que guio el presente estudio fue: ¿cómo es la inclusión de las investigadoras en los centros de investigación del IPN? Para responder esta interrogante fue necesario conocer cuál es el nivel de estudios del personal académico en centros de investigación del IPN; cuál es su distribución por horas de contratación; cuántos investigadores e investigadoras obtienen becas, estímulos o licencias y quienes acceden más ellas; cuántos son miembros del SNI; cómo es la distribución de los proyectos de investigación por área y tipo de investigación y cuál es la proporción de participación de mujeres y hombres; cuál es la participación en las redes de investigación y posgrado del IPN; cuál es la participación de la comunidad politécnica en materia de género, y cómo se encuentran conformados los colegios de profesores de los centros de investigación del IPN. 


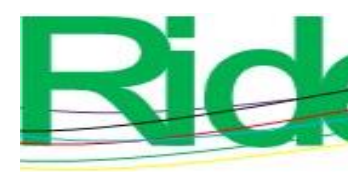

Revista Iberoamericana para la Investigación y el Desarrollo Educativo ISSN $2007-7467$

\section{Método}

El objetivo general de la investigación fue encontrar y analizar datos cuantitativos que muestren las disimetrías de las investigadoras en los centros de investigación del IPN. La importancia del estudio radica en mostrar evidencias que demuestren desigualdades de perspectiva de género en los centros de investigación del instituto en cuestión. El diseño de la investigación es no experimental y transeccional. La metodología fue cuantitativa con enfoque exploratorio y descriptivo.

El primer paso fue seleccionar la muestra. De la población de 20 centros de investigación científica y tecnológica del IPN, únicamente se seleccionaron 18. Los centros que no se consideraron para el estudio fueron: el Centro de Nanociencias y Micro y Nanotecnologías, porque no cuenta con una sección de investigación y posgrado, y el Centro de Investigación y de Estudios Avanzados, porque es un organismo público descentralizado con personalidad jurídica y patrimonio propios, además de contar con 28 departamentos de investigación que se encuentran distribuidos en nueve planteles a lo largo de la república mexicana, por dichas característica, pues, este centro requeriría un estudio distinto.

El segundo paso fue dividir la investigación en dos partes. La primera parte consistió en recabar los datos secundarios, los cuales debieron ser indagados y reunidos para su análisis estadístico, después fue necesario categorizarlos y finalmente posicionarlos como indicadores para fundamentar la pregunta de investigación. La segunda parte de la investigación consistió en obtener los datos primarios. Esta información fue recolectada especialmente para su examinación. Las cifras se obtuvieron de los colegios de profesores de los centros de investigación del IPN. Para recopilar los datos, se examinó de manera meticulosa su integración, haciendo énfasis en el número de investigadoras que los conforman. La información fue documentada de cada una de las páginas electrónicas de los 18 centros de investigación científica y tecnológica. 


\section{Resultados}

El personal académico del IPN está conformado 16380 docentes, de los cuales 9986 son hombres y 6394 son mujeres. La mayor cantidad del personal se encuentra en el nivel superior y posgrado, seguido por el nivel medio superior, después está el área central, enseguida se encuentran los centros de investigación y, al final, los centros de educación continua (IPN, 2018, p. 28). En la figura 1 se puede ver la distribución de personal académico por áreas en el IPN, así como la cantidad de hombres y mujeres.

Figura 1. Distribución de personal académico por áreas en el IPN

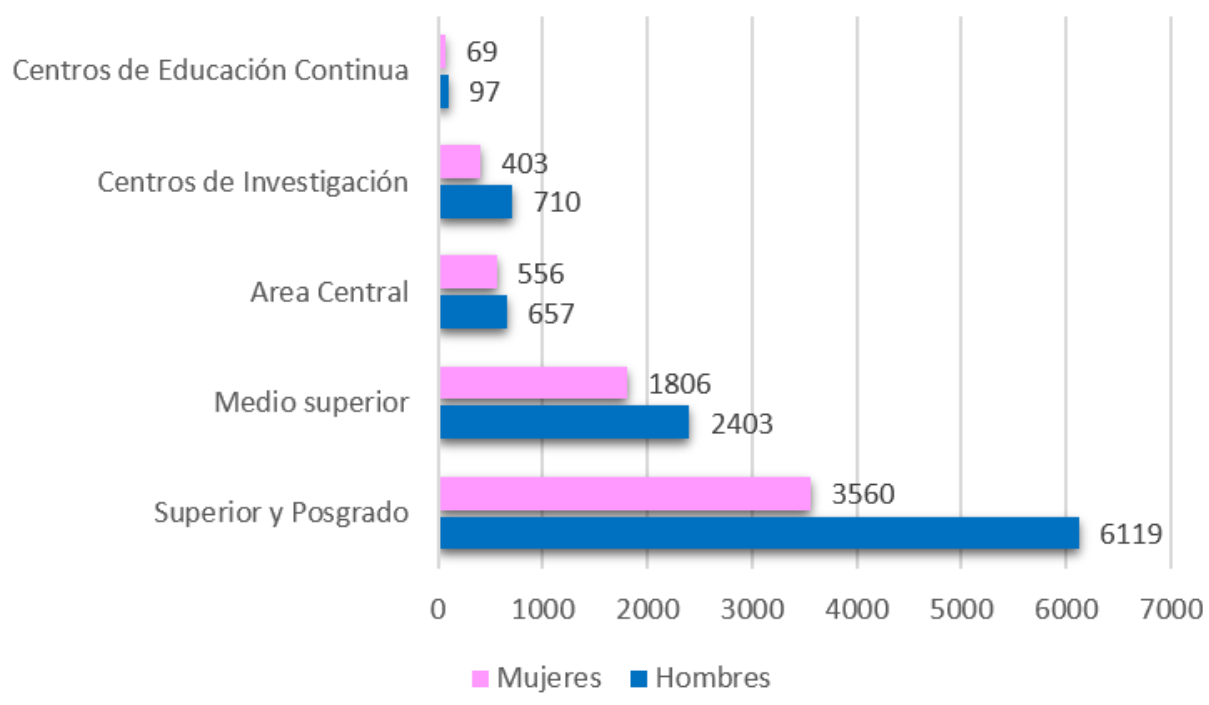

Fuente: Elaboración propia

La figura 1 evidencia que en los centros de investigación del IPN se encuentran adscritos 1113 docentes, de los cuales 710 son hombres y 403 son mujeres. Los datos anteriores nos permiten respaldar la afirmación de que el número de investigadoras es menor a la cantidad de investigadores.

Referente al nivel de estudios del personal académico en centros de investigación del IPN, se encontró que la escolaridad del personal masculino es mayor que el personal femenino en todos los grados (IPN, 2018, p. 28). En la figura 2 se puede observar esta tendencia. 


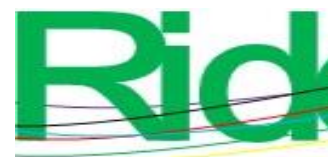

\section{Revista Iberoamericana para la Investigación y el Desarrollo Educativo ISSN 2007 - 7467}

Figura 2. Personal académico por nivel de estudios en centros de investigación del IPN

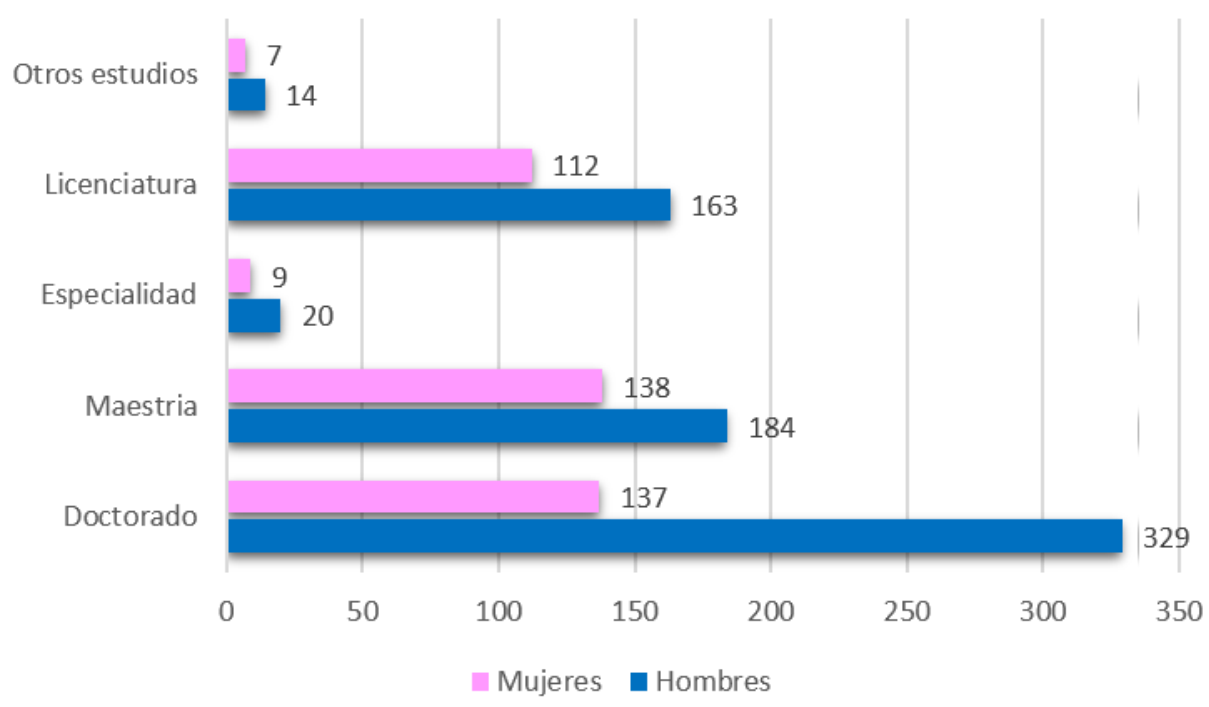

Fuente: Elaboración propia

Por otra parte, el personal académico del IPN es contratado por tiempo completo (40 horas), por tres cuartos de tiempo (30 horas), por medio tiempo (20 horas) y por horas (de una a 19 horas) (IPN, 2018, p. 29). Su distribución en los centros de investigación se puede ver en la figura 3.

Figura 3. Personal académico de centros de investigación por horas contractuales

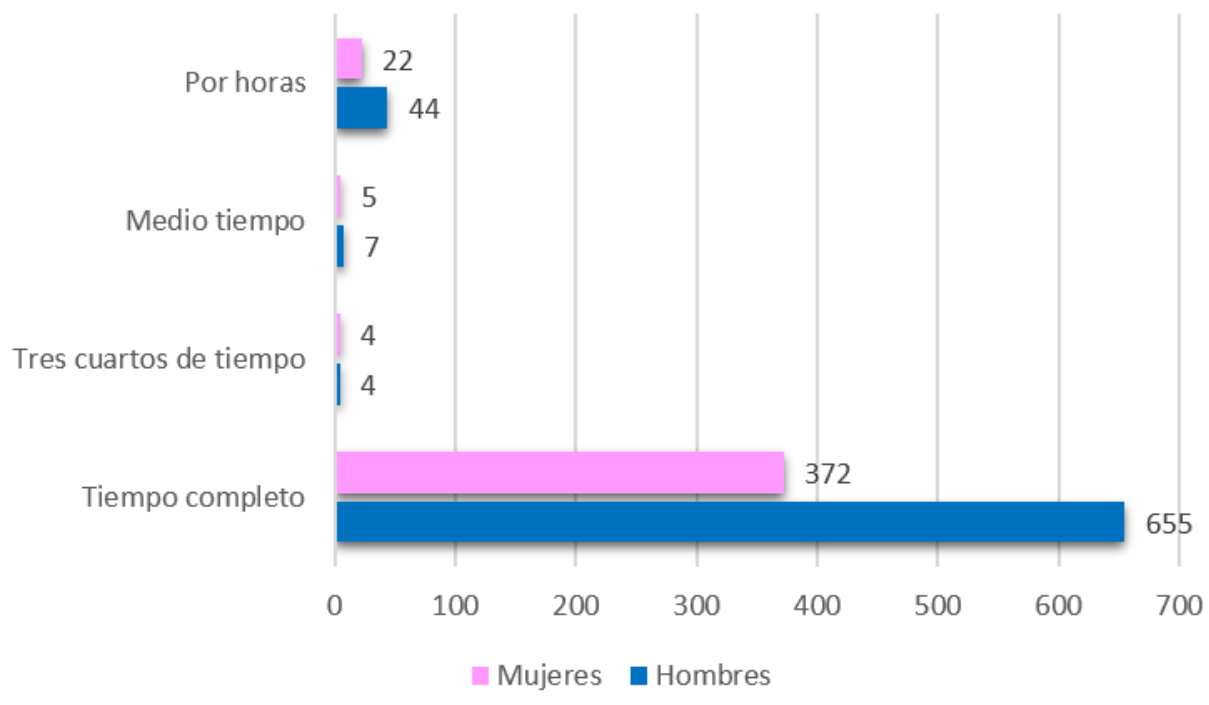

Fuente: Elaboración propia 

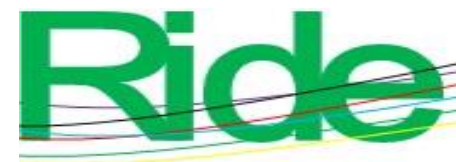

Revista Iberoamericana para la Investigación y el Desarrollo Educativo

ISSN 2007 - 7467

Las cantidades plasmadas en la figura 3 ponen en claro una vez más que el personal masculino predomina en todos los apartados, a excepción del rubro Tres cuartos de tiempo, donde las cantidades son semejantes.

Con respecto a las becas y estímulos que otorga el IPN, se encuentran las becas de exclusividad de la Comisión de Operación y Fomento de Actividades Académicas, el Estímulo al Desempeño de los Investigadores, el Estímulo al Desempeño Docente y las Licencias con Goce de Sueldo (IPN, 2018, p. 30). En la figura 4 se pueden analizar las cantidades correspondiente a estos rubros.

Figura 4. Becas, estímulos y licencias al personal académico de centros de investigación

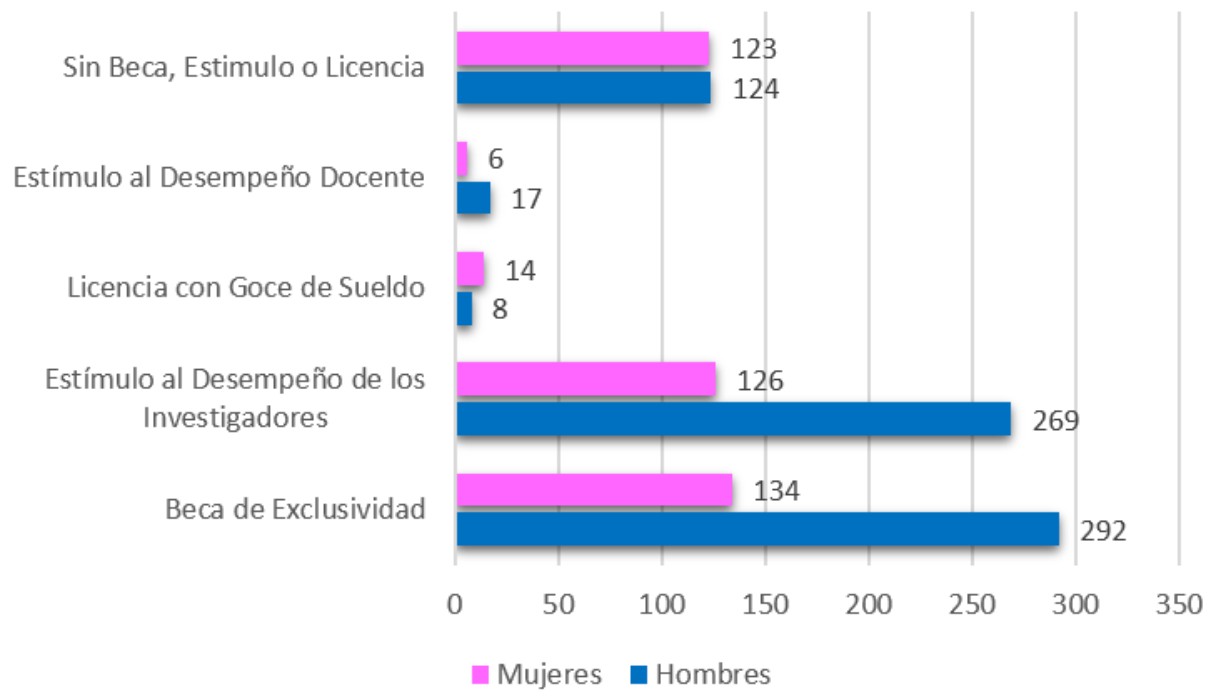

Fuente: Elaboración propia

Los datos de la figura 4 determinan que el apartado de Licencias con Goce de Sueldo es el único que registra más mujeres que hombres; en todos los demás el personal masculino sobresale por encima de $50 \%$. Para una mejor apreciación de los datos, se incluyó a los investigadores sin becas, estímulos o licencias.

En relación con el personal académico que son miembros del SNI, la proporción de hombres y mujeres indica que la presencia de investigadoras es menor y disminuye gradualmente conforme más alto es el nivel (IPN, 2018, p. 55). Esta tendencia se puede verificar en la figura 5. 


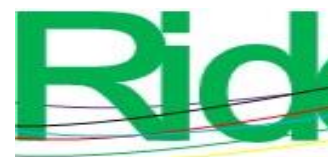

\section{Revista Iberoamericana para la Investigación y el Desarrollo Educativo ISSN 2007 - 7467}

Figura 5. Académicos de centros de investigación miembros del SNI en el año 2018

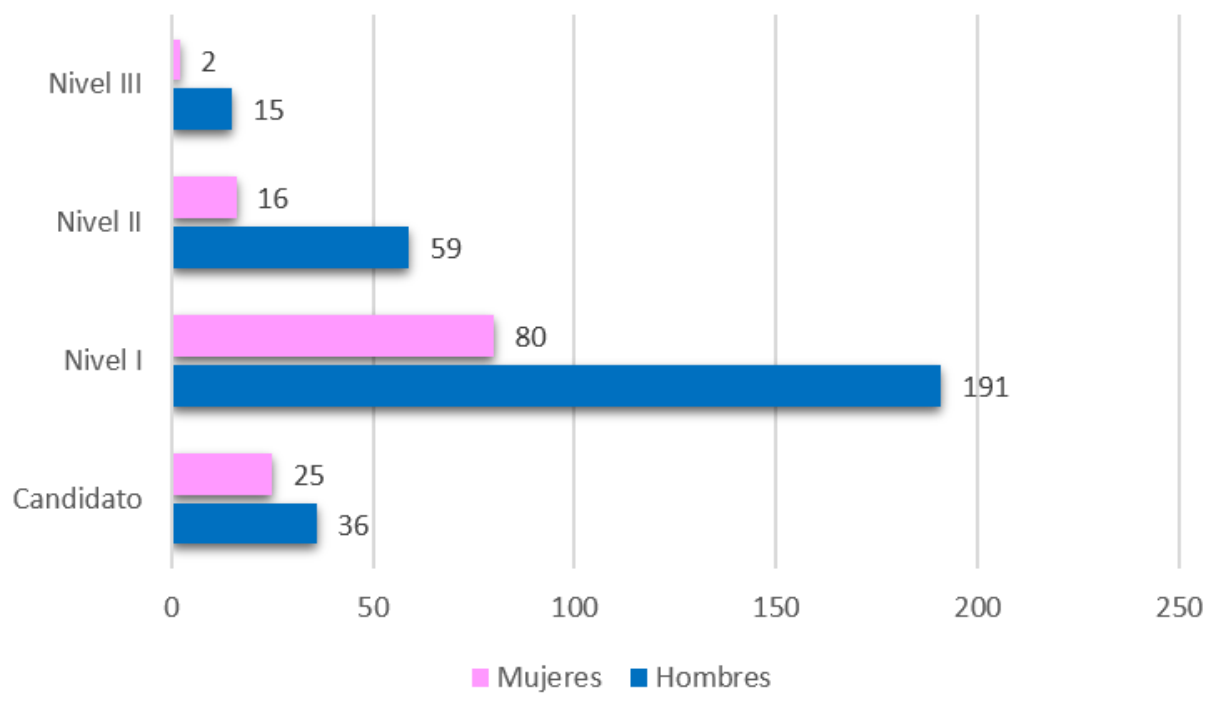

Fuente: Elaboración propia

Concerniente a la distribución de proyectos de investigación por área en centros de investigación, el IPN financió 1779 proyectos de investigación en el año 2018, de los cuales 610 fueron asignados a centros de investigación. Su distribución por área de investigación fue la siguiente: en ingeniería y tecnología 251, en ciencias naturales 155, en ciencias agrícolas 127, en ciencias sociales 32, en ciencias médicas 26, en educación 17 y en humanidades dos; estos datos se pueden observar en la figura 6 .

Figura 6. Proyectos de investigación IPN por área en centros de investigación

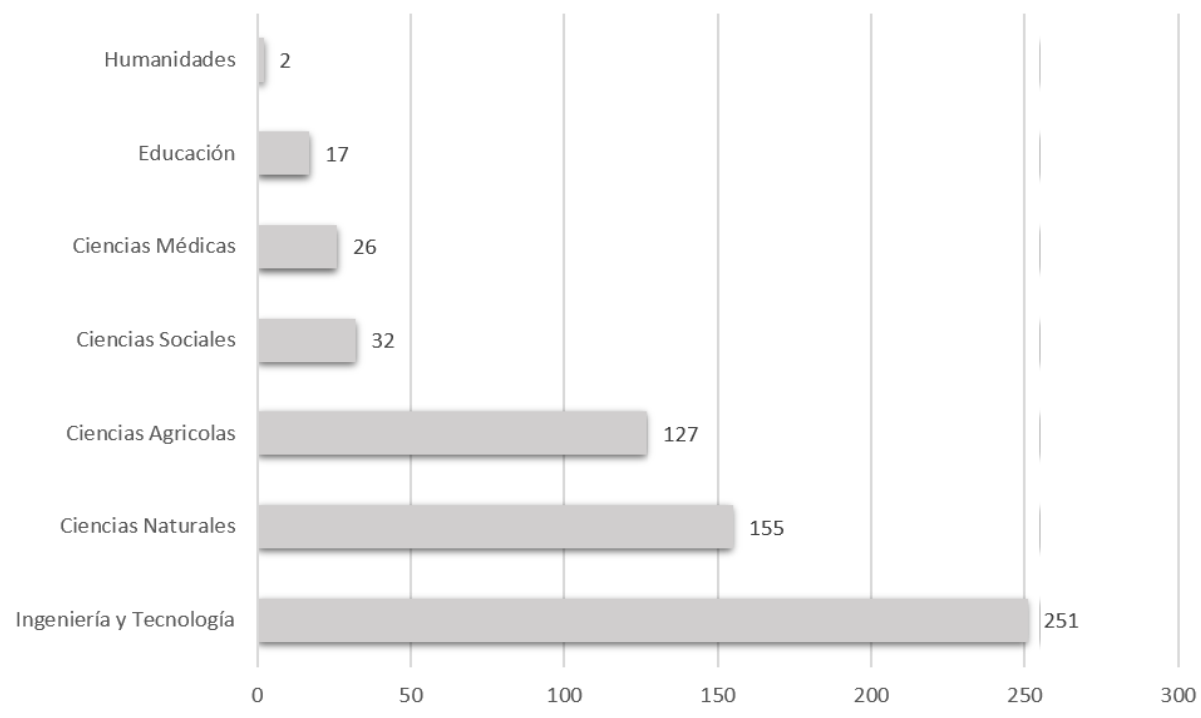

Fuente: Elaboración propia 

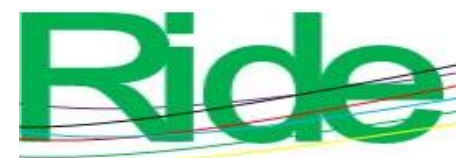

Revista Iberoamericana para la Investigación y el Desarrollo Educativo

ISSN $2007-7467$

Respecto su ordenamiento por tipo de investigación, en la figura 7 se puede ver las cantidades que destacan en esta tendencia. La mayor parte de los proyectos se realizan en investigación básica (40.3\%), en aplicada (33.4\%) y en desarrollo tecnológico (23.2 \%) (IPN, 2018, p. 53).

Figura 7. Proyectos por tipo de investigación en centros de investigación

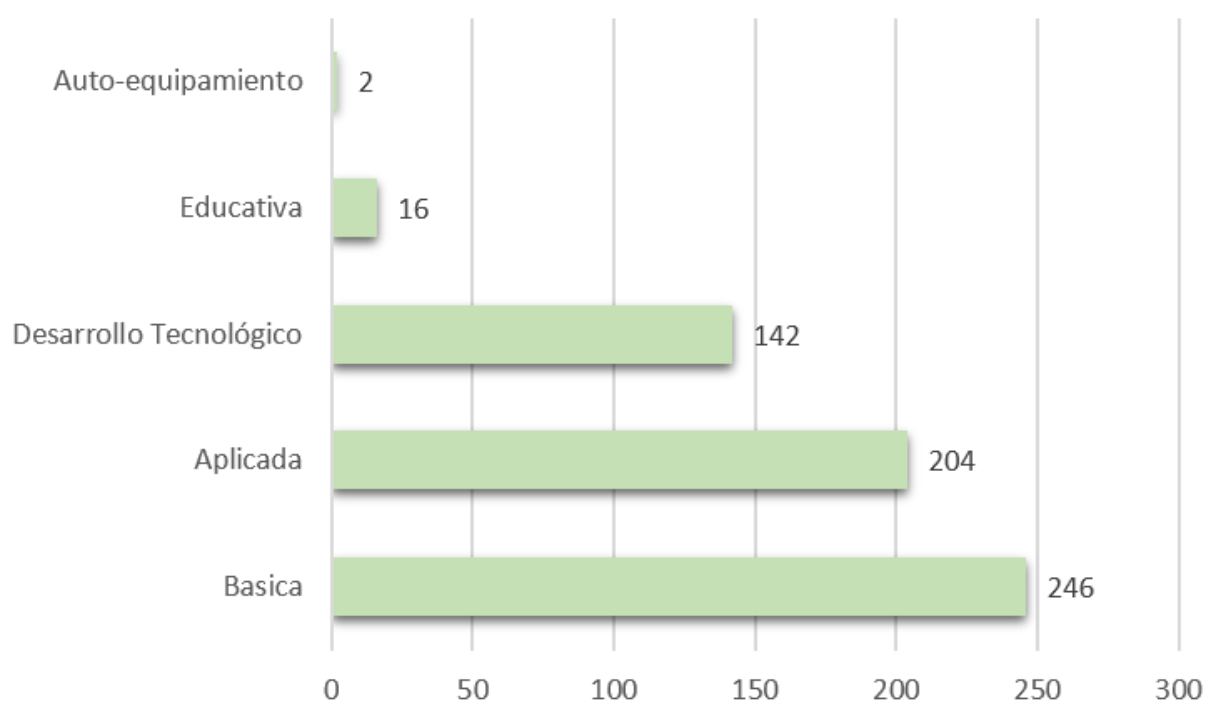

Fuente: Elaboración propia

Para Peinado, Cerecedo y Jaramillo (2015), "la gestión de la producción científica en el IPN es una integración de diversos elementos que intervienen en este proceso" (p. 148). Acerca de los participantes en proyectos de investigación del IPN, esto es, acerca de las y los investigadores que contribuyen con su conocimiento y experiencia en los proyectos de investigación, en específico en los asignados a los centros de investigación, sin aludir únicamente a los directores de los proyecto, sino a todos los que colaboran en él; en estos proyectos de investigación, decíamos, participó un total de 2258 investigadores: 1365 hombres y 893 mujeres (60.4\% y $39.5 \%$, respectivamente) (IPN, 2018, p. 54). Estos datos se muestran en la figura 8. Como se puede apreciar, existe una menor participación de investigadoras en proyectos de investigación. 
Figura 8. Participantes en proyectos de los centros de investigación

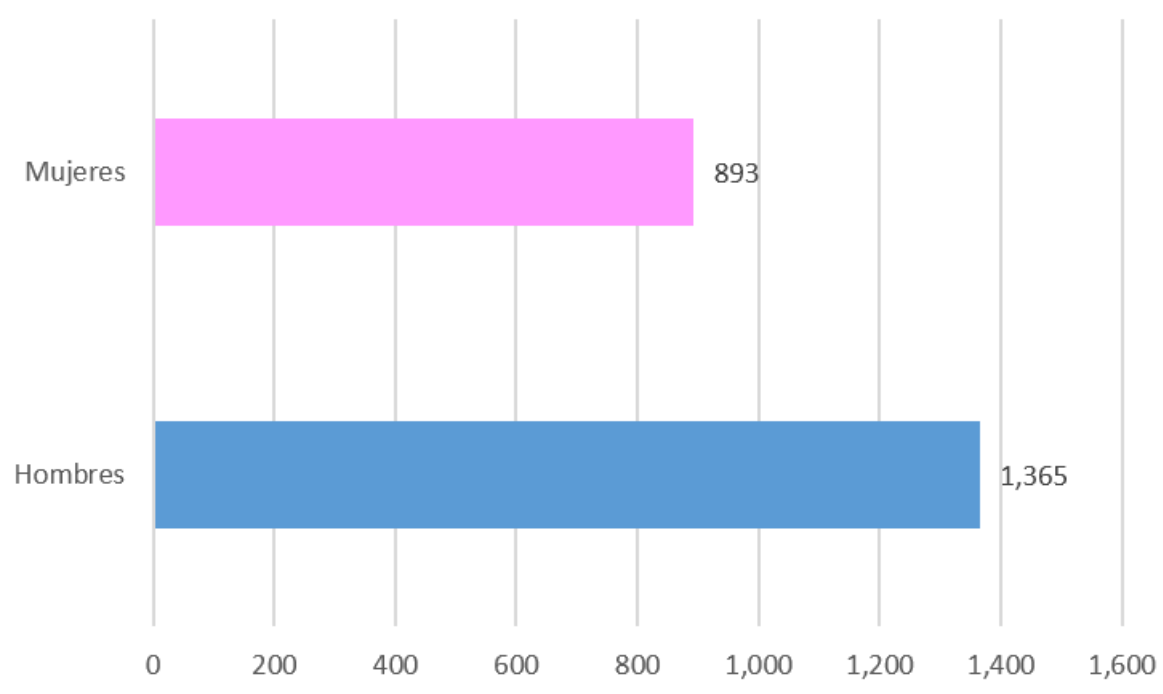

Fuente: Elaboración propia

\section{Redes de investigación y posgrado en el IPN}

Otro punto que vale la pena mencionar son las redes de investigación y posgrado del IPN, las cuales se crearon a partir del 30 de noviembre de 2006 como órganos de asesoría, consulta, apoyo y coordinación de esta casa de estudios, con la finalidad de promover la formación de recursos humanos de excelencia académica y profesional, así como la generación de conocimientos científicos de vanguardia y su transformación en aplicaciones útiles a la sociedad en dicha materia. En otras palabras, es la asociación de investigadores o personas que colaboran y aportan sus conocimientos y habilidades en el impulso sinérgico de programas de posgrado y proyectos de investigación en red (IPN, 2011, p. 2). En las nueve redes constituidas en el IPN participan 619 investigadores, 379 hombres y 240 mujeres (IPN, 2018, p. 57). En la figura 9 se describe el número de participantes en cada red. 

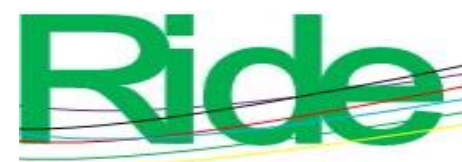

Revista Iberoamericana para la Investigación y el Desarrollo Educativo ISSN 2007 - 7467

Figura 9. Participantes en redes de investigación y posgrado en el IPN

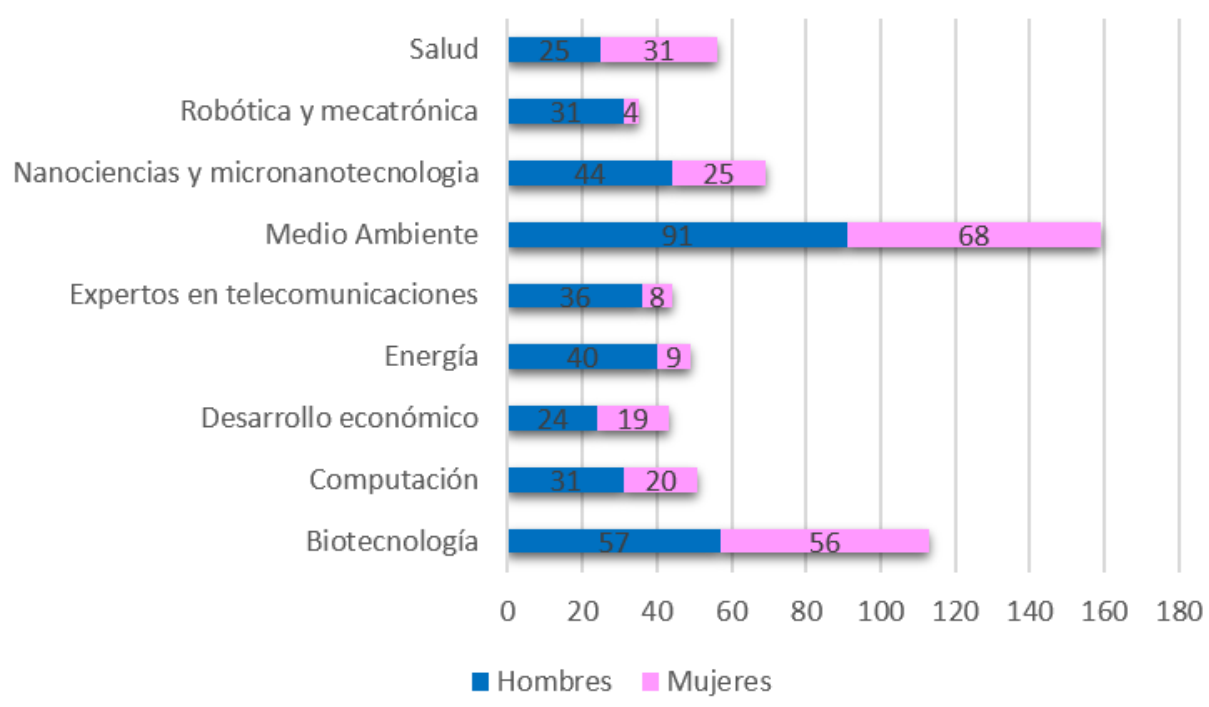

Fuente: Elaboración propia

La figura 9 permite ver que la mayor participación de investigadoras se realiza en la redes Biotecnología y Medio ambiente, con 56 y 68 respectivamente. Las redes con menor participación de investigadoras son Energía con nueve, Robótica y mecatrónica con cuatro y Expertos en telecomunicaciones con ocho. Esta figura, además de indicarnos la baja participación de investigadoras en redes de investigación, también permite ver en qué áreas se puede fortalecer su participación.

\section{Participación de la comunidad del IPN en materia de género}

Desde el año 2012, el IPN constituyó redes de género en todas sus escuelas, centros, unidades y área central, con el objetivo de contribuir en la integración del enfoque de género en el quehacer cotidiano del IPN, así como a las estrategias de seguimiento e impacto en la comunidad politécnica. Las redes de género están conformadas por un conjunto de personas interrelacionadas que desempeñan diversas acciones con la finalidad de contribuir en la integración del enfoque de género en el quehacer institucional. Precisan un papel fundamental para favorecer una cultura de igualdad y buen trato en los espacios educativos y laborales a través de actividades de sensibilización, capacitación, difusión y promoción (IPN, 2012, p. 17). En el año 2018, las redes de género estuvieron conformadas por 466 participantes, 310 mujeres y 156 hombres; no es difícil señalar la escasa colaboración del 


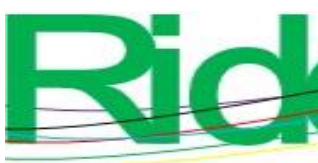

Revista Iberoamericana para la Investigación y el Desarrollo Educativo

ISSN $2007-7467$

personal masculino en esta actividad, es el único rubro donde sobresale la participación de las mujeres (IPN, 2018, p. 125). Esta información se especifica en la tabla 1.

Tabla 1. Redes de género del IPN en el año 2018

\begin{tabular}{|l|c|c|c|c|}
\hline \multicolumn{1}{|c|}{ Nivel } & $\begin{array}{c}\text { Núm. de } \\
\text { redes }\end{array}$ & Mujeres & Hombres & Total \\
\hline Medio superior & 19 & 81 & 48 & 129 \\
\hline Superior y posgrado & 25 & 106 & 54 & 160 \\
\hline Centros de investigación & 7 & 20 & 14 & 34 \\
\hline Área central & 33 & 103 & 40 & 143 \\
\hline Total & 84 & 310 & 156 & 466 \\
\hline
\end{tabular}

Fuente: IPN (2018)

\section{Conformación de los colegios de profesores en los centros de investigación del IPN}

En palabras de Peinado y Jaramillo (2018), "una característica muy específica de los posgrados en el IPN es el trabajo colegiado que se efectúa en ellos” (p. 132). Los colegios de profesores del IPN son órganos académicos de asesoría conformados por los profesores de posgrado con nombramiento de colegiado de una unidad académica; tienen por objeto coadyuvar a las unidades académicas del IPN en el cumplimiento de sus funciones en materia de posgrado (IPN, 2017, p. 34). En los colegios de profesores se realiza el trabajo para el mejor desarrollo de los programas académicos respecto de las líneas de investigación, los procesos de admisión, planeación y evaluación de cursos, designación o cambios de director o directores de tesis, conformación y evaluación de comités tutoriales, comisiones revisoras de tesis, exámenes predoctorales y de grado (Peinado, 2020, p. 5), así como de las actividades necesarias para la difusión de la oferta de programas académicos, la solicitud de becas o apoyos para alumnos y profesores de posgrado, la organización de expedientes para la acreditación de la calidad de programas académicos, entre otras. Peinado, Mayagoitia y Cruz (2019) comentan que "el trabajo que se realiza en los colegios de profesores es una ventaja fundamental en el desarrollo y consolidación de los posgrados, es una práctica que se no debe 


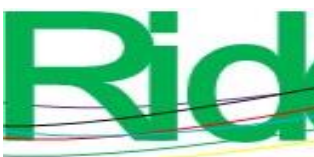

\section{Revista Iberoamericana para la Investigación y el Desarrollo Educativo ISSN 2007 - 7467}

dejar y en la que se deben redoblar esfuerzos para generar consensos y acuerdos que beneficien (p. 21)”. De ahí la importancia de su conformación y análisis para este estudio.

La recopilación de datos de los colegios de profesores de los centros de investigación del IPN se enfocó en examinar de manera general su integración, haciendo énfasis en el número de investigadoras que los conforman. En la tabla 2 se encuentra la descripción correspondiente.

Tabla 2. Conformación de los colegios de profesores por centro de investigación

\begin{tabular}{|c|c|c|c|c|}
\hline Centro de investigación & Mujeres & Hombres & Total & $\begin{array}{c}\text { \% de } \\
\text { mujeres }\end{array}$ \\
\hline $\begin{array}{l}\text { 1) Centro de Biotecnología } \\
\text { Genómica }\end{array}$ & 7 & 15 & 22 & $31.8 \%$ \\
\hline $\begin{array}{l}\text { 2) Centro de Innovación y Desarrollo } \\
\text { Tecnológico en Cómputo }\end{array}$ & 5 & 21 & 26 & $19.2 \%$ \\
\hline $\begin{array}{l}\text { 3) Centro de Investigación en } \\
\text { Biotecnología Aplicada }\end{array}$ & 25 & 21 & 46 & $54.3 \%$ \\
\hline $\begin{array}{l}\text { 4) Centro de Investigación en } \\
\text { Ciencia Aplicada y Tecnología } \\
\text { Avanzada Unidad Legaria }\end{array}$ & 17 & 36 & 53 & $32.0 \%$ \\
\hline $\begin{array}{l}\text { 5) Centro de Investigación en } \\
\text { Computación }\end{array}$ & 4 & 40 & 44 & $9.0 \%$ \\
\hline $\begin{array}{l}\text { 6) Centro de Investigaciones } \\
\text { Económicas, Administrativas y } \\
\text { Sociales }\end{array}$ & 13 & 16 & 29 & $44.8 \%$ \\
\hline $\begin{array}{l}\text { 7) Centro Interdisciplinario de } \\
\text { Investigación para el Desarrollo } \\
\text { Integral Regional Unidad Durango }\end{array}$ & 27 & 27 & 54 & $50 \%$ \\
\hline $\begin{array}{l}\text { 8) Centro Interdisciplinario de } \\
\text { Investigación para el Desarrollo } \\
\text { Integral Regional Unidad Oaxaca }\end{array}$ & 30 & 49 & 79 & $37.9 \%$ \\
\hline
\end{tabular}




\begin{tabular}{|c|c|c|c|c|}
\hline $\begin{array}{l}\text { 9) Centro Interdisciplinario de } \\
\text { Investigación y Estudios sobre } \\
\text { Medio Ambiente y Desarrollo }\end{array}$ & 10 & 20 & 30 & $33.3 \%$ \\
\hline $\begin{array}{l}\text { 10) Centro de Desarrollo de } \\
\text { Productos Bióticos }\end{array}$ & 19 & 15 & 34 & $55.8 \%$ \\
\hline $\begin{array}{l}\text { 11) Centro de Investigación e } \\
\text { Innovación Tecnológica }\end{array}$ & 6 & 14 & 20 & $30 \%$ \\
\hline $\begin{array}{l}\text { 12) Centro de Investigación en } \\
\text { Ciencia Aplicada y Tecnología } \\
\text { Avanzada Unidad Altamira }\end{array}$ & 1 & 10 & 11 & $9.0 \%$ \\
\hline $\begin{array}{l}\text { 13) Centro de Investigación en } \\
\text { Ciencia Aplicada y Tecnología } \\
\text { Avanzada Unidad Querétaro }\end{array}$ & 8 & 24 & 32 & $25 \%$ \\
\hline $\begin{array}{l}\text { 14) Centro de Investigación y } \\
\text { Desarrollo de Tecnología Digital }\end{array}$ & 4 & 22 & 26 & $15.3 \%$ \\
\hline $\begin{array}{l}\text { 15) Centro Interdisciplinario de } \\
\text { Ciencias Marinas }\end{array}$ & 14 & 48 & 62 & $22.5 \%$ \\
\hline $\begin{array}{l}\text { 16) Centro Mexicano para la } \\
\text { Producción más Limpia }\end{array}$ & 7 & 6 & 13 & $53.8 \%$ \\
\hline $\begin{array}{l}\text { 17) Centro Interdisciplinario de } \\
\text { Investigación para el Desarrollo } \\
\text { Integral Regional Unidad Michoacán }\end{array}$ & 7 & 15 & 22 & $31.8 \%$ \\
\hline $\begin{array}{l}\text { 18) Centro Interdisciplinario de } \\
\text { Investigación para el Desarrollo } \\
\text { Integral Regional Unidad Sinaloa }\end{array}$ & 13 & 29 & 42 & $30.9 \%$ \\
\hline Totales & 217 & 428 & 645 & $33.6 \%$ \\
\hline
\end{tabular}

\section{Fuente: Elaboración propia}

En relación con los porcentajes, el Centro de Investigación en Biotecnología Aplicada, el Centro Interdisciplinario de Investigación para el Desarrollo Integral Regional Unidad Durango, el Centro de Desarrollo de Productos Bióticos y el Centro Mexicano para la Producción más Limpia tienen 50 \% o más de investigadoras; los demás están por debajo de esta proporción. Los centros de investigación con menos investigadoras son: el Centro de 


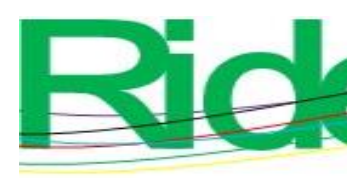

Revista Iberoamericana para la Investigación y el Desarrollo Educativo

ISSN 2007 - 7467

Investigación en Computación, 4 de 44, el Centro de Investigación en Ciencia Aplicada y Tecnología Avanzada Unidad Altamira, 1 de 11, el Centro de Investigación y Desarrollo de Tecnología Digital, 4 de 26, y el Centro de Investigación en Ciencia Aplicada y Tecnología Avanzada Unidad Querétaro, 8 de 32.

Es importante mencionar que los datos recabados en la tabla 2 incluyen profesores colegiados y de asignatura; no se consideraron los profesores invitados o visitantes. Cabe especificar que la página electrónica del Centro Mexicano para la Producción más Limpia no tiene referida esta sección, las cantidades fueron obtenidas de su directorio. Asimismo, debido a que algunas páginas no exhiben a detalle los currículos de los profesores, no fue posible elaborar un análisis más minucioso y considerar otros parámetros que permitieran resaltar las diferencias entre mujeres y hombres en la investigación, además de una diferenciación más exhaustiva entre cada centro, dependiendo, claro está, del área a la que pertenecen (ingeniería y ciencias físico matemáticas, ciencias médico biológicas, ciencias sociales y administrativas, e interdisciplinaria). Esta información puede ser el preámbulo para otras investigaciones más detalladas que permitan visualizar el trabajo de las mujeres investigadoras en los centros de investigación del IPN. Las cantidades no coinciden con los datos presentados inicialmente porque únicamente se recabó información de los colegios de profesores, y no del total del personal académico adscrito a cada centro de investigación.

\section{Discusión}

Los resultados obtenidos permitieron corroborar disimetrías de las investigadoras en los centros de investigación del IPN. El primer punto a observar es que esta problemática podría originarse en etapas de formación anteriores, lo que impacta directamente al momento de integrarse a un puesto de trabajo. Como menciona Nielsen (2017) en su estudio de caso sobre el fenómeno de la exclusión voluntaria entre las jóvenes investigadoras, "las investigadoras de carrera temprana se enfrentan a una serie de inconvenientes que producen opciones de carrera limitadas y evitan que algunas de ellas traduzcan con éxito sus capacidades en recompensas científicas suficientes y avance profesional” (p. 149).

Por otra parte, aunque se ha incrementado el número de mujeres en su ingreso a las universidades, se siguen formando pocas en la ciencia, especialmente en ciencias exactas. Esta podría ser la causa del menor número de investigadoras en los centros de investigación 


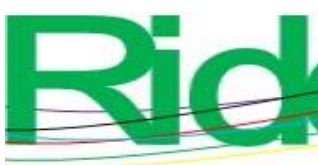

\section{Revista Iberoamericana para la
Investigación y el Desarrollo Educativo \\ ISSN 2007 - 7467}

del IPN y, consecuentemente, una cantidad inferior de investigadoras en el SNI. Aunado a lo anterior, las investigadoras que ingresan al SNI ascienden en menor medida que sus pares hombres en los niveles II y III (Izquierdo y Atristan, 2019, p. 128). Por ejemplo, en el año 2016 había 25072 miembros en el SNI, 15991 hombres (64 \%) y 9081 mujeres (36 \%). En el año 2017 había 27186 miembros del sistema, 17204 hombres (63\%) y 9982 mujeres (37\%), un incremento de 901 mujeres. Asimismo, $96 \%$ tenía estudios de doctorado y $3 \%$ de maestría (Consejo Nacional de Ciencia y Tecnología [Conacyt], 2017, pp. 57-63). Las mujeres representan $36.6 \%$ del total nacional de investigadores en el SNI, y se concentran en gran parte en los niveles I y Candidato (Izquierdo y Atristan, 2019, p. 128). En el caso específico del IPN, las estadísticas no son muy diferentes: los académicos miembros del SNI en el año 2018 fueron 809 hombres y 407 mujeres. En la tabla 3 se observan 56 hombres y 9 mujeres en el nivel III; 155 hombres y 55 mujeres en el nivel II; 486 hombres y 261 mujeres en el nivel I; 112 hombres y 82 mujeres en el nivel Candidato (IPN, 2018, p. 55).

Tabla 3. Académicos del IPN miembros SNI

\begin{tabular}{|c|c|c|c|c|c|c|c|c|c|c|c|}
\hline \multirow[t]{2}{*}{ Adscripción } & \multicolumn{2}{|c|}{ Candidato } & \multicolumn{2}{|c|}{ Nivel I } & \multicolumn{2}{|c|}{ Nivel II } & \multicolumn{2}{|c|}{$\begin{array}{c}\text { Nivel } \\
\text { III }\end{array}$} & \multicolumn{3}{|c|}{ Totales } \\
\hline & $\mathrm{H}$ & $\mathrm{M}$ & $\mathrm{H}$ & $\mathrm{M}$ & $\mathrm{H}$ & $\mathrm{M}$ & $\mathrm{H}$ & M & $\mathrm{H}$ & $\mathrm{M}$ & Tota \\
\hline Medio superior & 2 & 2 & 1 & 2 & 0 & 0 & 0 & 0 & 3 & 4 & 7 \\
\hline Superior y posgrado & 69 & 51 & $\begin{array}{r}27 \\
9\end{array}$ & $\begin{array}{r}17 \\
5\end{array}$ & 96 & $\begin{array}{l}3 \\
9\end{array}$ & 41 & 7 & $\begin{array}{r}48 \\
5\end{array}$ & $\begin{array}{r}27 \\
2\end{array}$ & 757 \\
\hline $\begin{array}{l}\text { Centros de } \\
\text { investigación }\end{array}$ & 36 & 25 & $\begin{array}{r}19 \\
1\end{array}$ & 80 & 59 & $\begin{array}{l}1 \\
6\end{array}$ & 15 & 2 & $\begin{array}{r}30 \\
1\end{array}$ & $\begin{array}{r}12 \\
3\end{array}$ & 424 \\
\hline Área central & 5 & 4 & 15 & 4 & 0 & 0 & 0 & 0 & 20 & 8 & 28 \\
\hline Totales & 112 & 82 & $\begin{array}{r}48 \\
6\end{array}$ & $\begin{array}{r}26 \\
1\end{array}$ & $\begin{array}{r}15 \\
5\end{array}$ & $\begin{array}{l}5 \\
5\end{array}$ & 56 & 9 & $\begin{array}{r}80 \\
9\end{array}$ & $\begin{array}{r}40 \\
7\end{array}$ & 1216 \\
\hline
\end{tabular}

Fuente: IPN (2018)

Referente a los colegios de profesores de los centros de investigación del IPN, el número de mujeres es menor que el de los hombres. Aunque los porcentajes de inclusión se han ido incrementando, no son equiparables para lograr la equidad de género que se requiere. Es imperativo crear estrategias, programas, planes, políticas o modelos que incentiven la 


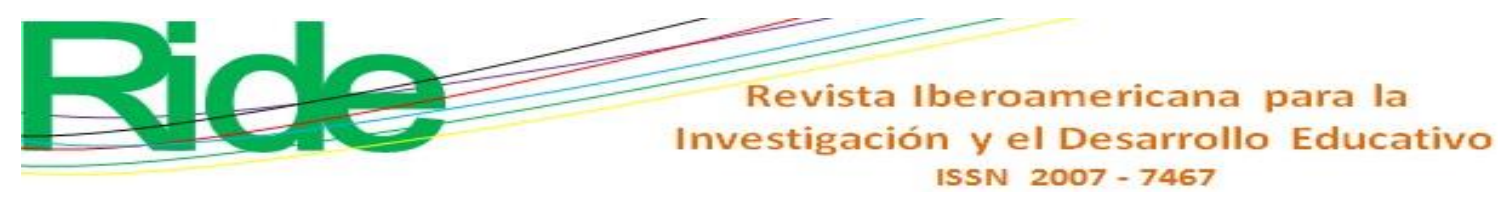

inclusión de más mujeres en el campo de la investigación, ya que, de acuerdo con Cárdenas (2015), "al aumentar la cantidad de mujeres en las categorías profesionales y en los equipos de trabajo del sector científico y tecnológico se pueden tener diferentes visiones y enriquecer la solución de los problemas" (p. 8). Incrementar el número de investigadoras es un tema de inclusión, equidad, perspectiva de género y de justicia social; es parte de la respuesta para generar más conocimiento y encontrar soluciones a problemas nacionales con impacto social y que beneficien el desarrollo del país.

Si bien hay varios estudios de perspectiva de género que se han realizado en las escuelas y en la academia (Buquet, 2011, p. 220; McKnight, 2018, p. 220; Román, Domínguez, Saucedo y Tánori, 2016, p. 84), también los centros de investigación son un espacio propicio para realizarlos (Vayreda, Conesa, Revelles y González, 2019, p. 430; Peinado, Montoy y Torres, 2020, p. 13), ya que su sensibilización motiva políticas de igualdad en las instituciones (Litosseliti, Gill y García, 2019, p. 1; Tapia, 2017, p. 7). En ese sentido, se han promovido prácticas normativas para disponer de cierto porcentaje, para la inclusión de las mujeres en varios campos de la vida diaria. En la comunidad académica y de investigación se podría implementar esta práctica con el afán de que las investigadoras dispongan de espacios suficientes para su crecimiento y desarrollo. Esto beneficiaría a todos, porque de lo que se trata es ofrecer equidad a todas y a todos los miembros de la sociedad.

Es necesario aceptar que prevalecen múltiples obstáculos en la vida de las investigadoras: el uso del tiempo, responsabilidad de la familia, maternidad, disparidad en la inserción laboral, condiciones de trabajo, estereotipos de género, cultura organizacional, entre otros. Sin embargo, de acuerdo con Mattsson (2015), "las mujeres en colectividad experimentan cierta uniformidad que las hace fuertes como grupo y se adaptan mejor en el mundo académico" (p. 685). Macoun y Miller (2014), a su vez, determinan que "las redes de apoyo informales entre pares pueden proporcionar vías para el sostenimiento y sustento en el mundo académico" (p. 287). La acumulación de estas ventajas les permite disminuir tensiones con las normas de la academia (Jenkins, 2014, p. 262; Sang, 2018, p. 192; Peinado, 2020, p. 15); de esta manera, los retos y las adversidades que enfrentan sirven para vislumbrar el éxito en sus funciones (Redmond, Gutke, Galligan, Howard y Newman, 2017, p. 332).

Finalmente, es preciso señalar que, si bien el presente estudio encontró evidencias cuantitativas de las disimetrías que impactan en las investigadoras, entre sus limitaciones se puede señalar que no es generalizable o concluyente (Hernández, Fernández y Baptista, 2010, 


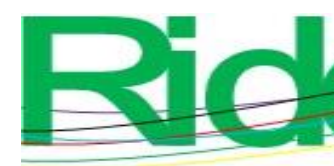

Revista Iberoamericana para la Investigación y el Desarrollo Educativo

ISSN 2007 - 7467

p. 165). Es completamente necesario seguir investigando en este tema, así como estimular el crecimiento y fortalecimiento de esta línea de investigación. Para futuros trabajos se recomienda extender el análisis a otros centros e institutos de investigación públicos y privados, ya sea por separado o de manera conjunta. También se recomienda ampliar la dimensión de este tópico, incluyendo experiencias de las docentes investigadoras y de los docentes investigadores, indagar sobre prácticas de los directivos y el impacto de este fenómeno en el marco normativo y en el ámbito económico.

\section{Conclusiones}

El objetivo de la investigación fue encontrar y analizar datos cuantitativos que muestren las disimetrías de las investigadoras en los centros de investigación del IPN. Las estadísticas aquí presentadas son más significativas de lo que parecen. La comunidad académica se encuentra ante una gran dificultad, los esfuerzos requeridos para resolver estas disimetrías son muy exigentes, pero existe la mejor disponibilidad para superarlos.

Los datos reafirman el reflejo de las asimetrías que enfrentan las investigadoras. Necesariamente se reconoce la intervención de otras variables antes y durante su formación. El antes se manifiesta en sus roles como mujer y la tradición de enfocarse solamente en determinados campos de estudio que intervienen directamente con su formación. El después, por las decisiones que tiene que tomar en función de su compleja situación de mujer-madreesposa, la cual se contrapone en gran medida a la académica-investigadora, sobre todo si se toma en cuenta que en los claustros de investigación aún hoy en día prevalecen prácticas arcaicas de control y sumisión.

Sin pretender que esto parezca una justificación, se necesita tiempo para absorber todos los planteamientos de las investigadoras, que ciertamente son entendibles, necesarios y urgentes; se requiere tiempo para concluir con una tendencia de costumbres equivocadas, malos hábitos y pensamientos desviados que se precisan olvidar para formar las nuevas generaciones, porque son en ellas en las que se debe trabajar con mayor ahínco desde todos los ámbitos, contextos y perspectivas posibles. 


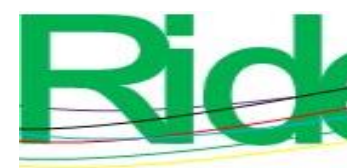

Revista Iberoamericana para la Investigación y el Desarrollo Educativo ISSN $2007-7467$

\section{Referencias}

Aiston, S. and Jung, J. (2015). Women academics and research productivity: An international comparison. Gender and Education, 27(3), 205-220. Retrieved from https://doi.org/10.1080/09540253.2015.1024617.

Blanco, R. (2018). Del fulgor al desencanto. Desafíos para el feminismo académico en la fugaz experiencia de la Carrera de Estudios de la Mujer (Universidad de Buenos Aires). Revista Interdisciplinaria de Estudios de Género de El Colegio de México, 4(7), 1-31. Recuperado de http://dx.doi.org/10.24201/eg.v4i0.159.

Blazquez, N. y Bustos, O. (2013). Saber y poder. Testimonios de directoras de la UNAM. (1. ${ }^{\mathrm{a}}$ ed.). Ciudad de México, México: Centro de Investigaciones Interdisciplinarias en Ciencias y Humanidades.

Buquet, A. (2011). Transversalización de la perspectiva de género en la educación superior: Problemas conceptuales y prácticos. Perfiles educativos, 33(especial), 211-225. Recuperado de https://www.iisue.unam.mx/perfiles/numeros/2011/e.

Bustos, B. (2017). Profesionales, trayectorias y uso del tiempo. Egresadas de la Universidad de Guadalajara. La Ventana. Revista de Estudios de Género, 5(45), 269-305. Recuperado de http://revistalaventana.cucsh.udg.mx/index.php/LV/article/view/5058.

Cárdenas, M. (2015). La participación de las mujeres investigadoras en México. Investigación Administrativa, 44(116), 1-23. Recuperado de https://www.ipn.mx/assets/files/investigacionadministrativa/docs/revistas/116/art4.pdf

Castellano, D. (2 de julio de 2019). Sesgos de género alejan de licenciaturas y posgrados a mujeres adolescentes: expertos. La Jornada. Recuperado de https://www.jornada.com.mx/2019/07/02/sociedad/035n1soc.

Consejo Nacional de Ciencia y Tecnología [Conacyt]. (2017). Informe general del estado de la ciencia, la tecnología y la innovación México 2017. México: Consejo Nacional de $\begin{array}{llll}\text { Ciencia } & \text { y } & \text { Tecnologíal } & \text { Recuperado }\end{array}$ http://www.siicyt.gob.mx/index.php/transparencia/informes-conacyt/informegeneral-del-estado-de-la-ciencia-tecnologia-e-innovacion/informe-general2017/4813-informe-general-2017/file. 


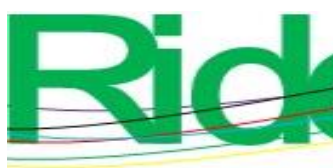

\section{Revista Iberoamericana para la Investigación y el Desarrollo Educativo ISSN $2007-7467$}

Estrada, J., Mendieta, A. y González, B. (2016). Perspectiva de género en México: Análisis de los obstáculos y limitaciones. Opción, 32(13), 12-36. Recuperado de http://produccioncientificaluz.org/index.php/opcion/article/view/21587.

García, M. (2014). Reflexiones sobre los retos que enfrentan las mujeres en el ingreso, la permanencia y la promoción en el sistema nacional de investigadores. Revista de Comunicación de la SEECI, 35(18), 18-25. Recuperado de http://dx.doi.org/10.15198/seeci.2014.35E.18-25.

Gamboa, F. y Pérez, A. (2017). Tiempo de academia y el poder 'poder' de las mujeres en el desafío familia-trabajo. Las académicas de la Universidad Michoacana de San Nicolás de Hidalgo. La Ventana. Revista de Estudios de Género, 5(45), 241-268.

Recuperado de http://revistalaventana.cucsh.udg.mx/index.php/LV/article/view/5370.

Gómez, B. y Tena, O. (2018). Narrativas de mujeres en torno a su experiencia de no maternidad: resistencias ante tecnologías de género. Revista Interdisciplinaria de Estudios de Género de El Colegio de México, 4(10), 1-35. Recuperado de http://dx.doi.org/10.24201/eg.v4i0.310.

Gupta, N. (2016). Perceptions of the Work Environment: The Issue of Gender in Indian Scientific Research Institutes. Indian Journal of Gender Studies, 23(3), 437-466. Retrieved from https://doi.org/10.1177/0971521516656079.

Heiskanen, T., Otonkorpi, K., Leinonen, M. and Ylöstalo, H. (2018). Gender issues on change agenda-Practising intersectionality in action research. Gender, Work \& Organization, 25(5), 459-474. Retrieved from https://onlinelibrary.wiley.com/doi/10.1111/gwao.12072.

Hernández, R., Fernández, C. y Baptista, P. (2010). Metodología de la investigación (1. a ed.). México: McGraw-Hill.

Hoskins, K. (2015). Researching female professors: the difficulties of representation, positionality and power in feminist research. Gender and Education, 27(4), 393-411. Retrieved from https://doi.org/10.1080/09540253.2015.1021301.

Instituto Politécnico Nacional [IPN]. (2011). Lineamientos para la creación y operación de redes de investigación y posgrado. Gaceta Politécnica, 14(48), 1-8. Recuperado de https://www.ipn.mx/assets/files/normatividad/docs/Lineamientos/gac_885.pdf. 


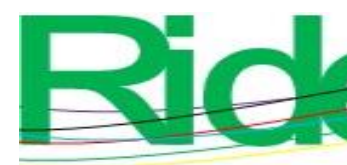

Revista Iberoamericana para la Investigación y el Desarrollo Educativo

ISSN 2007 - 7467

Instituto Politécnico Nacional [IPN]. (2012). Acuerdo por el que se crea la unidad politécnica de gestión con perspectiva de género. Gaceta Politécnica, 15(41), 17-20. Recuperado de

https://www.aplicaciones.abogadogeneral.ipn.mx/ACUERDOS/acuerdos_ipn_4/GA C_EXT_932B_unidad_pol_gest_persp_gen.pdf.

Instituto Politécnico Nacional [IPN]. (2017). Reglamento de estudios de posgrado. Gaceta Politécnica, 18(54), 34-58. Recuperado de https://www.ipn.mx/assets/files/normatividad/docs/reglamentos/GACEXT1358.pdf.

Instituto Politécnico Nacional [IPN]. (2018). Informe anual de actividades 2018. Ciudad de México, México: Instituto Politécnico Nacional. Recuperado de https://www.ipn.mx/informeanual2018.pdf.

Instituto Politécnico Nacional [IPN]. (2020). Reglamento de Orgánico del Instituto Politécnico Nacional. Gaceta Politécnica, 18(56), 3-65. Recuperado de https://www.ipn.mx/assets/files/ccs/docs/gaceta-extraordinaria/2020/03/reglamento1541.pdf

Izquierdo, I. y Atristan, M. (2019). Experiencias de investigadoras en su ingreso, promoción y permanencia en el Sistema Nacional de Investigadores: tensiones y estrategias identitarias. IE Revista de Investigación Educativa de la REDIECH, 10(18), 127-142. Recuperado de http://dx.doi.org/10.33010/ie_rie_rediech.v10i18.466

Jabbaz, M., Samper, T. y Díaz, C. (2019). La brecha salarial de género en las instituciones científicas. Estudio de caso. Convergencia Revista de Ciencias Sociales, 26(80), 127. Recuperado de_https://doi.org/10.29101/crcs.v26i80.11248.

Jenkins, K. (2014). "That's not philosophy": feminism, academia and the double bind. Journal of Gender Studies, 23(3), 262-274. Retrieved from https://doi.org/10.1080/09589236.2014.909720

Kannen, V. (2013). Pregnant, privileged and PhDing: exploring embodiments in qualitative research. Journal of Gender Studies, 22(2), 178-191. Retrieved from https://doi.org/10.1080/09589236.2012.745681

Litosseliti, L., Gill, R. and García, L. (2019). Postfeminism as a critical tool for gender and language study. Gender \& Language, 13(1), 1-22. Retrieved from https://journals.equinoxpub.com/index.php/GL/article/view/34599 


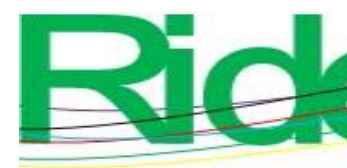

\section{Revista Iberoamericana para la Investigación y el Desarrollo Educativo ISSN $2007-7467$}

Macías, G. e Islas, C. (2018). Las mujeres y la sociedad del conocimiento: uso y aplicabilidad de las TIC por las investigadoras. La Ventana. Revista de Estudios de Género, 6(48), 208-235.

Recuperado

de http://www.revistalaventana.cucsh.udg.mx/index.php/LV/article/view/6662

Macoun, A. and Miller, D. (2014). Surviving (thriving) in academia: Feminist support networks and women ECRs. Journal of Gender Studies, 23(3), 287-301. Retrieved from https://doi.org/10.1080/09589236.2014.909718

Mattsson, T. (2015). "Good girls": emphasised femininity as cloning culture in academia. Gender and Education, 27(6), 685-699. Retrieved from https://doi.org/10.1080/09540253.2015.1069796

McDonald, J. (2016). Occupational Segregation Research: Queering the Conversation. Gender, Work and Organization, 23(1), 19-35. Retrieved from https://doi.org/10.1111/gwao.12100

McKnight, L. (2018). A bit of a dirty word: "feminism" and female teachers identifying as feminist. Journal of Gender Studies, 27(2), 220-230. Retrieved from https://doi.org/10.1080/09589236.2016.1202816

Meza M., Galbán, S. y Ortega C. (2019). Experiencias y retos de las mujeres pertenecientes al Sistema Nacional de Investigadores. RIDE Revista Iberoamericana para la Investigación y el Desarrollo Educativo, 10(19). Recuperado de https://doi.org/10.23913/ride.v10i19.491

Moreno, M. (2018). Trayectorias educativas de las mujeres universitarias: efectos de los roles de género en el retorno al sistema educativo. La Ventana. Revista de Estudios de Género, 5(47), 139-177. Recuperado de http://www.revistalaventana.cucsh.udg.mx/index.php/LV/article/view/6707

Nielsen, M. (2017). Reasons for Leaving the Academy: a Case Study on the 'Opt Out' Phenomenon among Younger Female Researchers. Gender, Work \& Organization, 24(2), 134-155. Retrieved from https://doi.org/10.1111/gwao.12151

Nikunen, M. and Lempiäinen, K. (2018). Gendered strategies of mobility and academic career. Gender and Education, 32(4), 554-571. Retrieved from https://doi.org/10.1080/09540253.2018.1533917 


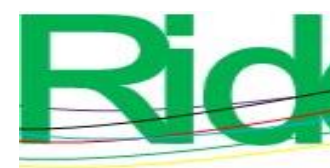

Revista Iberoamericana para la
Investigación y el Desarrollo Educativo
ISSN $2007-7467$

Peinado, J., Cerecedo, M. y Jaramillo, D. (2015). Propuesta de un modelo de gestión del Capital Intelectual para los Centros de Investigación del IPN. Punto de vista, 6(10), 135-157. Recuperado de http://dx.doi.org/10.15765/pdv.v6i10.768

Peinado, J. y Jaramillo, D. (2018). La eficiencia terminal del Centro de Investigación e Innovación Tecnológica. Revista Electrónica de Investigación Educativa, 20(3), 126134. Recuperado de https://doi.org/10.24320/redie.2018.20.3.1797

Peinado, J., Mayagoitia, V. y Cruz, C. (2019). Los grupos de investigación y su impacto en los factores que determinan la eficiencia terminal. Revista Dilemas Contemporáneos: Educación, Política y Valores, 7(1), 1-26. Recuperado de https://doi.org/10.46377/dilemas.v28i1.1712

Peinado, J., Montoy, L. y Torres, Z. (2020). Estrategia de gestión para la generación de recursos en un centro de investigación y posgrado. Estudio de caso del CIITEC en el contexto de México. Acta Universitaria, 30(e2445), 1-25. Recuperado de https://doi.org/10.15174/au.2020.2445

Peinado, J. (2020). Factores vinculados en la selección de aspirantes de maestría y doctorado. Estudio de caso de un centro de investigación y posgrado. Revista Dilemas contemporáneos: Educación, Política y Valores, $\quad 8(1), \quad$ 1-25. https://doi.org/10.46377/dilemas.v8i1.2395

Román, R., Domínguez, S., Saucedo, M. y Tánori, J. (2016). Validación de un instrumento sobre cultura de género en instituciones de educación, salud e investigación en el noroeste de México. La Ventana. Revista de Estudios de Género, 5(44), 83-109. Recuperado de http://revistalaventana.cucsh.udg.mx/index.php/LV/article/view/6010

Redmond, P., Gutke, H., Galligan, L., Howard, A. and Newman, T. (2017). Becoming a female leader in higher education: investigations from a regional university. Gender and Education, 29(3), 332-351. Retrieved from https://doi.org/10.1080/09540253.2016.1156063

Sang, K. (2018). Gender, ethnicity and feminism: An intersectional analysis of the lived experiences feminist academic women in UK higher education. Journal of Gender $\begin{array}{llll}\text { Studies, } & \text { 27(2), } & \text { 192-206. } & \text { Retrieved }\end{array}$ https://doi.org/10.1080/09589236.2016.1199380 

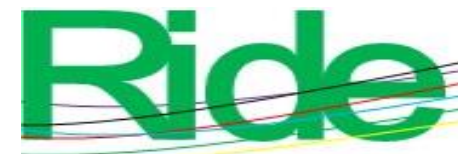

Sinha, S. (2015). More educated and more equal? A comparative analysis of female education and employment in Japan, China and India. Gender and Education, 27(7), 846-870. Retrieved from https://doi.org/10.1080/09540253.2015.1103367

Tapia, G. (2017). Graciela Hierro: Filosofía de la educación en clave de género. Revista Interdisciplinaria de Estudios de Género de El Colegio de México, 3(5), 1-22. Recuperado de https://estudiosdegenero.colmex.mx/index.php/eg/article/view/94/86

Vayreda, A., Conesa, E., Revelles, B. and González, A. (2019). Subjectivation processes and gender in a neoliberal model of science in three Spanish research centres. Gender, Work and Organization, 26(4), 430-447. Retrieved from https://doi.org/10.1111/gwao.12360

Zippel, K. (2019). Gendered images of international research collaboration. Gender, Work \& Organization, 26(12), 1794-1805. Retrieved form https://doi.org/10.1111/gwao.12233 